\title{
Momentum Conservative Schemes for Shallow Water Flows
}

\author{
S. R. Pudjaprasetya* and I. Magdalena \\ Industrial and Financial Mathematics Research Group, Faculty of Mathematics \\ and Natural Sciences, Institut Teknologi Bandung, Jalan Ganesha 10, Bandung \\ 40132, Indonesia.
}

Received 29 September 2013; Accepted (in revised version) 17 March 2014

Available online XXX

\begin{abstract}
We discuss the implementation of the finite volume method on a staggered grid to solve the full shallow water equations with a conservative approximation for the advection term. Stelling \& Duinmeijer [15] noted that the advection approximation may be energy-head or momentum conservative, and if suitable which of these to implement depends upon the particular flow being considered. The momentum conservative scheme pursued here is shown to be suitable for 1D problems such as transcritical flow with a shock and dam break over a rectangular bed, and we also found that our simulation of dam break over a dry sloping bed is in good agreement with the exact solution. Further, the results obtained using the generalised momentum conservative approximation for 2D shallow water equations to simulate wave run up on a conical island are in good agreement with benchmark experimental data.
\end{abstract}

AMS subject classifications: 65M08, 76M12, 35L65

Key words: Finite volume method, staggered grid, conservative scheme, shallow water equations.

\section{Introduction}

Shallow water equations (SWE) are often used to model fluid flow in rivers, lakes, estuaries or coastal areas. The main assumption in adopting a SWE model is that the horizontal length scale is much greater than the vertical scale - and for example, complex changes to rapidly varying flows in coastal hydrodynamics may be described, including the inundation of dry land. From the mathematical point of view, the mass and momentum conservation equations involved constitute an hyperbolic system, and the finite volume method is known to be very effective for computations involving equations derived from conservation laws (such as in SWE modelling).

${ }^{*}$ Corresponding author. Email addresses:

ikha.magdalena@yahoo.com (I. Magdalena)

sr_pudjap@math.itb.ac.id (S R. Pudjaprasetya), 
Unlike finite difference methods, where derivatives at a point are replaced with truncated Taylor series, in finite volume methods the focus is on approximating the conservative equations in discrete form on a fixed volume in space called a cell. The flux entering a given cell is identified with that leaving an adjacent cell or cells, so finite volume methods are known as conservative schemes. In Godunov, Suliciu or other relaxation schemes, the two equations in the SWE are approximated on the same cell and the numerical fluxes are computed using an approximate Riemann solver — cf. $[2,10,14,17,18]$ for descriptions and analysis of this approach. Refs. $[8,13,19]$ present thorough investigations of a high-order finite volume scheme applied to uniform or non-uniform meshes for the SWE model. Alternatively, finite volume schemes can involve approximations on adjacent cells, resulting in schemes where the two unknowns of the SWE are calculated on a staggered grid. Implementing a finite volume scheme on a staggered grid has a great advantage, for the numerical fluxes can be computed more simply since the need to use an approximate Riemann solver can be avoided. Staggered finite volume approximations for solving nonlinear hyperbolic systems involving conservation laws have been investigated in the past few years $[4,9,15,16]$. The advection term in a SWE model is the most difficult to approximate, and Stelling \& Duinmeijer [15] discuss two alternative approaches (energy-head or momentum conservative) - although their applicability depends upon the particular problem. The appropriate choice of conservative method for topographies with abrupt changes has been discussed [15],

In this article, we show that the momentum conservative approach is suitable for dam break simulation for various bottom topographies - and also for simulations of 2D wave run up on a conical island, found to be in good agreement with experimental data [3] previously used for validating various nonlinear shallow water equations solvers $[6,12,20]$. Our discussion starts in Section 2 with a description of the finite volume method on a staggered grid for a simple linear SWE model. The momentum conservative scheme for a nonlinear 1D SWE model is then discussed in Section 3, and in Section 4 the scheme is implemented for various shallow water flows. The analogous conservative scheme for 2D shallow water equations is described in Section 5, and in Section 6 we present the simulation of run up waves on a conical island and compare the experimental data of Ref. [3].

\section{Finite Volume Method on a Staggered Grid for a Simple Linear SWE Model}

We first discuss the formulation of a leapfrog method for a linear SWE model, and stress its equivalence with the finite volume method on a staggered grid. This provides a solid building block for further development since the method is explicit, non-dissipative, and conditionally stable.

The simple governing equations for small amplitude gravity waves above a flat bottom $d_{0}$ are

$$
\begin{aligned}
& \eta_{t}+d_{0} u_{x}=0, \\
& u_{t}+g \eta_{x}=0,
\end{aligned}
$$


where $\eta(x, t)$ denotes the surface elevation measured from the undisturbed water level, $u(x, t)$ the horizontal component of water velocity, and $g$ is the constant gravitational acceleration.

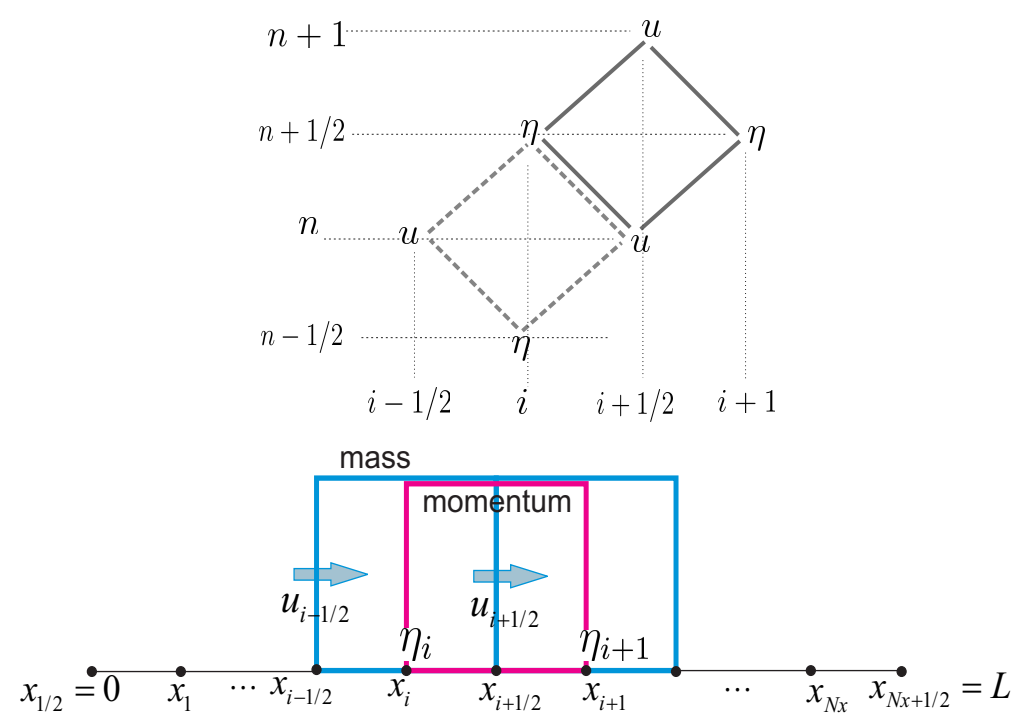

Figure 1: (Top) Leapfrog stencil (Bottom) Illustration of staggered grid, with a cell for mass and a cell for momentum.

In a spatial domain $0<x<L$ with hard wall left and right boundary conditions $u(0, t)=u(L, t)=0$, we define a staggered numerical grid with partition points $x_{1 / 2}=$ $0, x_{1}, \cdots, x_{i-1 / 2}, x_{i}, x_{i+1 / 2}, \cdots, x_{N x+1 / 2}=L$, and apply the second order leapfrog scheme to (2.1) around grid points $\left(x_{i}, t_{n}\right)$, and to (2.2) around $\left(x_{i+1 / 2}, t_{n+1 / 2}\right)$, with stencils as depicted in Fig. 1 (Top). Thus we consider the scheme

$$
\begin{aligned}
& \frac{\eta_{i}^{n+1 / 2}-\eta_{i}^{n-1 / 2}}{\Delta t}+d_{0} \frac{u_{i+1 / 2}^{n}-u_{i-1 / 2}^{n}}{\Delta x}=0, \\
& \frac{u_{i+1 / 2}^{n+1}-u_{i+1 / 2}^{n}}{\Delta t}+g \frac{\eta_{i+1}^{n+1 / 2}-\eta_{i}^{n+1 / 2}}{\Delta x}=0 .
\end{aligned}
$$

or equivalently

$$
\begin{aligned}
& \frac{\eta_{i}^{n+1}-\eta_{i}^{n}}{\Delta t}+d_{0} \frac{u_{i+1 / 2}^{n}-u_{i-1 / 2}^{n}}{\Delta x}=0, \\
& \frac{u_{i+1 / 2}^{n+1}-u_{i+1 / 2}^{n}}{\Delta t}+g \frac{\eta_{i+1}^{n+1}-\eta_{i}^{n+1}}{\Delta x}=0 .
\end{aligned}
$$

Equations (2.5) and (2.6) can be interpreted as a finite volume approximation of the linear SWE (2.1) and (2.2). Thus consider an interval $0<x<L$ with a staggered grid as depicted in Fig. 1 (Bottom). This figure illustrates both the mass cell $\left[x_{i-1 / 2}, x_{i+1 / 2}\right]$ centred at the grid point $x_{i}$ where the mass conservation equation (2.1) is approximated, and 
the adjacent cell $\left[x_{i}, x_{i+1}\right]$ centred at the staggered grid point $x_{i+1 / 2}$ where the momentum conservation equation (2.2) is approximated. Eq, (2.5) is a discrete approximation of the mass conservation equation (2.1) in the cell $\left[x_{i-1 / 2}, x_{i+1 / 2}\right]$, since during time $\Delta t$ the change of mass in that cell corresponds to the difference between the mass flux through the sides $x_{i-1 / 2}$ and $x_{i+1 / 2}$, and in the linear SWE model the surface elevation $\eta$ is neglected such that these mass fluxes at the sides $x_{i-1 / 2}$ and $x_{i+1 / 2}$ are respectively $d_{0} u_{i-1 / 2}$ and $d_{0} u_{i+1 / 2}$. In respect of (2.6), the surface height difference between sides $x_{i}$ and $x_{i+1}$ of the cell $\left[x_{i}, x_{i+1}\right]$ with length $\Delta x$ yields an hydrostatic pressure difference $g\left(\eta_{i+1}^{n+1}-\eta_{i}^{n+1}\right)$, so the gravity induces an horizontal flow in that cell with rate $\left(u_{i+1 / 2}^{n+1}-u_{i+1 / 2}^{n}\right) / \Delta t$.

The leapfrog scheme (2.5) and (2.6) is of second-order accuracy, and the CourantFriedrichs-Lewy stability condition is $0<C \leq 1$ where $C=\sqrt{g d_{0}} \Delta t / \Delta x$. When dealing with large gradient solutions, the scheme suffers from weak dispersion error, but this does not occur for long waves. Finally, we note that this scheme is explicit and therefore very efficient, and exhibits no numerical damping error for any Courant number $C$ satisfying the stability condition.

\section{Momentum Conservative Scheme for a 1D Nonlinear SWE Model}

Consider the nonlinear shallow water equations written in conservative form

$$
\begin{aligned}
& h_{t}+(h u)_{x}=0, \\
& (h u)_{t}+\left(h u^{2}+\frac{1}{2} g h^{2}\right)_{x}=g h d_{x}-C_{f}|u| u,
\end{aligned}
$$

where $h(x, t)$ denotes the total water height, $\eta(x, t)$ the surface elevation, $u(x, t)$ the horizontal fluid velocity, $d(x)$ the bottom topography and $C_{f}$ the friction coefficient $-\mathrm{cf}$. Fig. (2). A familiar equivalent form of this system is

$$
\begin{aligned}
& h_{t}+(h u)_{x}=0, \\
& u_{t}+u u_{x}+g \eta_{x}=-C_{f} \frac{|u| u}{h},
\end{aligned}
$$

that can be obtained through a relation for the advection term

$$
u u_{x}=\frac{1}{h}\left(\frac{\partial(q u)}{\partial x}-u \frac{\partial q}{\partial x}\right)
$$

where $q=h u$ denotes the horizontal momentum. (This relation (3.5) will subsequently be used in approximating the advection term.)

We now proceed to describe our staggered grid finite volume scheme for Eqs. (3.3) and (3.4). The mass conservative approximation of Eq. (3.3) at the cell $\left[x_{i-1 / 2}, x_{i+1 / 2}\right]$ is

$$
\frac{d h_{i}^{n}}{d t}+\frac{{ }^{*} h_{i+\frac{1}{2}}^{n} u_{i+\frac{1}{2}}^{n}-{ }^{*} h_{i-\frac{1}{2}}^{n} u_{i-\frac{1}{2}}^{n}}{\Delta x}=0
$$




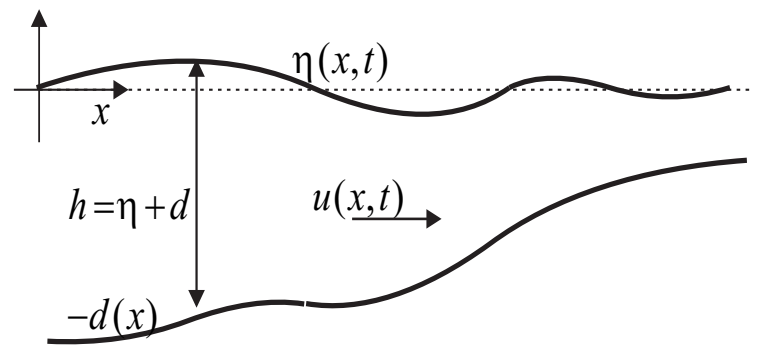

Figure 2: Sketch and notations.

- cf. also Fig. 3. Eq. (3.6) holds at every full grid point $x_{i}$, but must be approximated at half-step points denoted by $i+1 / 2$ (terms indicated by the superscript ${ }^{*}$ ). On implementing the first-order upwind approximation

$$
{ }^{*} h_{i+\frac{1}{2}}= \begin{cases}h_{i}, & \text { if } u_{i+1 / 2} \geq 0 \\ h_{i+1}, & \text { if } u_{i+1 / 2}<0\end{cases}
$$

the term ${ }^{*} h_{i+1 / 2} u_{i+1 / 2}$ expresses the first-order approximation of mass flux at the edge $x_{i+1 / 2}$ as shown in Fig. 3. The upwind approximation (3.7) is a direct consequence of considering the flow direction - when the flow is to the right $\left(u_{i+1 / 2} \geq 0\right)$, we take the left flux $h_{i} u_{i+1 / 2}$; and when the flow is to the left $\left(u_{i+1 / 2}<0\right)$, we take the right flux $h_{i+1} u_{i+1 / 2}$. Eq. (3.6) for positive flow is thus

$$
\frac{h_{i}^{n+1}-h_{i}^{n}}{\Delta t}+\frac{h_{i}^{n} u_{i+\frac{1}{2}}^{n}-h_{i-1}^{n} u_{i-\frac{1}{2}}^{n}}{\Delta x}=0 .
$$

Hence approximation (3.6) preserves mass conservation property at every cell $\left[x_{i-1 / 2}, x_{i+1 / 2}\right]$, and this holds for either flow direction.

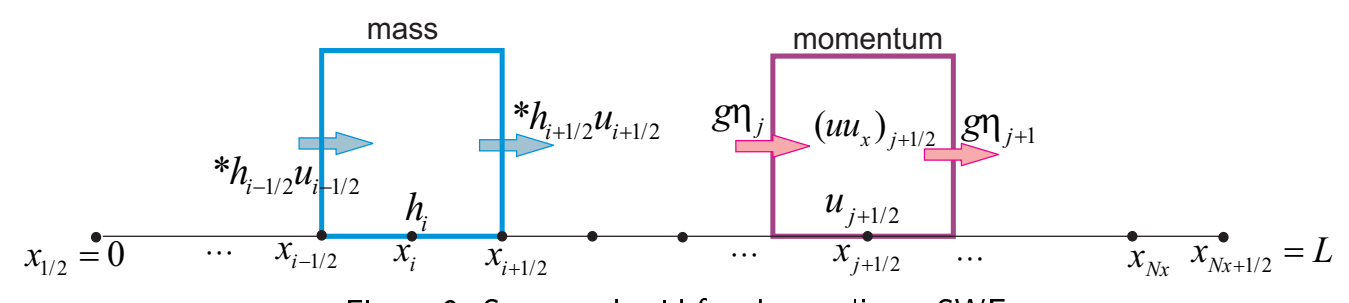

Figure 3: Staggered grid for the nonlinear SWE.

Analogous to Eq. (2.6), the approximated momentum equation (3.4) at the cell $\left[x_{i}, x_{i+1}\right]$ is

$$
\frac{d u_{i+1 / 2}^{n}}{d t}+\left(u u_{x}\right)_{i+1 / 2}^{n}+g \frac{\eta_{i+1}^{n+1}-\eta_{i}^{n+1}}{\Delta x}=-\left.C_{f} \frac{|u| u}{h}\right|_{i+1 / 2} ^{n}
$$

as Fig. 3 also illustrates, and the nonlinear friction term involved may be calculated using Picard linearisation - i.e.

$$
(|u| u)^{n} \approx\left|u^{n}\right| u^{n+1}
$$


The approximation of the advection term $\left(u u_{x}\right)_{i+1 / 2}$ is most important, and we recall for clarity the momentum conservative approximation introduced in Ref. [15] in approximating the advection term using the relation (3.5). Thus under the condition that that total water depth remains positive $\bar{h}_{i+1 / 2}>0$, the advection term in Eq. (3.5) is approximated by

$$
\begin{gathered}
\left(u u_{x}\right)_{i+1 / 2}=\frac{1}{\bar{h}_{i+\frac{1}{2}}}\left(\frac{\bar{q}_{i+1} * u_{i+1}-\bar{q}_{i}{ }^{*} u_{i}}{\Delta x}-u_{i+\frac{1}{2}} \frac{\bar{q}_{i+1}-\bar{q}_{i}}{\Delta x}\right), \\
\bar{h}_{i+1 / 2}=\frac{1}{2}\left(h_{i}+h_{i+1}\right), \quad \bar{q}_{i}=\frac{1}{2}\left(q_{i+\frac{1}{2}}+q_{i-\frac{1}{2}}\right), \quad q_{i+\frac{1}{2}}={ }^{*} h_{i+\frac{1}{2}} u_{i+\frac{1}{2}} .
\end{gathered}
$$

At points denoted by $i$ there is no value of $u$, as indicated by ${ }^{*} u_{i}$ and approximated by

$$
{ }^{*} u_{i}= \begin{cases}u_{i-\frac{1}{2}}, & \text { if } \bar{q}_{i} \geq 0, \\ u_{i+\frac{1}{2}}, & \text { if } \bar{q}_{i}<0 .\end{cases}
$$

Thus the choice similarly depends on the flow direction, as in approximating * $h$ via (3.7), and for positive flow the advection approximation is

$$
\frac{\bar{q}_{i}}{\bar{h}_{i+1 / 2}}\left(\frac{u_{i+\frac{1}{2}}-u_{i-\frac{1}{2}}}{\Delta x}\right)=\frac{h_{i} u_{i+\frac{1}{2}}+h_{i-1} u_{i-\frac{1}{2}}}{h_{i}+h_{i+1}}\left(\frac{u_{i+\frac{1}{2}}-u_{i-\frac{1}{2}}}{\Delta x}\right) .
$$

In summary, the spatial approximation for the nonlinear SWE model is to adopt (3.6) and (3.7) for mass conservation and (3.8), (3.9) and (3.10) for momentum balance. In Ref. [15], this approximation is called the momentum conservative scheme. It is secondorder accurate for the linear parts but only of order one for the nonlinear parts, since they are computed using the upwind approximation. We also note that Eq. (3.8) is implemented when the corresponding cell $\left[x_{i}, x_{i+1}\right]$ is wet, indicated by $h_{i}>0$ or $h_{i+1}>0$, whereas in dry cells the values $u_{i+1 / 2}$ are set to zero. This is known as the wet-dry procedure, allowing simulation of flows with dry areas.

\section{Test Simulations for the 1D Nonlinear SWE Model}

In this section, we discuss our implementation of the momentum conservative scheme for simulating several shallow water flows, and several other cases commonly used as benchmark tests for numerical SWE solvers. We find that our momentum conservative scheme produces results that compare well with those from other conservative schemes, and where there an exact solution we obtain good agreement. Except for a possible adjustment at the left and right boundaries, no special treatment required for all three examples below, indicating the robustness of our scheme. (For further comparisons, see Ref [7].)

\subsection{Transcritical flow with a shock.}

The following is a test case designed for a relatively long computational time before a steady state is reached $[2,11,21]$. We take the computational domain to be $0<x<25$ 
and consider shallow flow over the topography

$$
d(x)= \begin{cases}-0.2+0.05(x-10)^{2}, & \text { if } 8<x<12 \\ 0, & \text { otherwise }\end{cases}
$$

The initial data are $h(x, 0)=0.33$ and $u(x, 0)=0.18 / 0.33$, the left discharge boundary condition $h u(0, t)=0.18$, right boundary $h(25, t)=0.33$, and gravitational acceleration $g=9.81$. This test case contains flows with different Froude number $\operatorname{Fr}=u / \sqrt{g h}-$ i.e. $\mathrm{Fr}<1$ upstream and downstream but $\mathrm{Fr}>1$ above the bump. We used $\Delta x=0.05$ corresponding to $\mathrm{Nx}=501$ spatial grid points, and the time step $\Delta t=0.008$. The surface profile reached steady state around the time $t=125$ seconds and then remained steady, with the computation capturing a surface with a shock - cf. Fig. 4. Our result is plotted together with a reference result obtained using 2501 grid points.
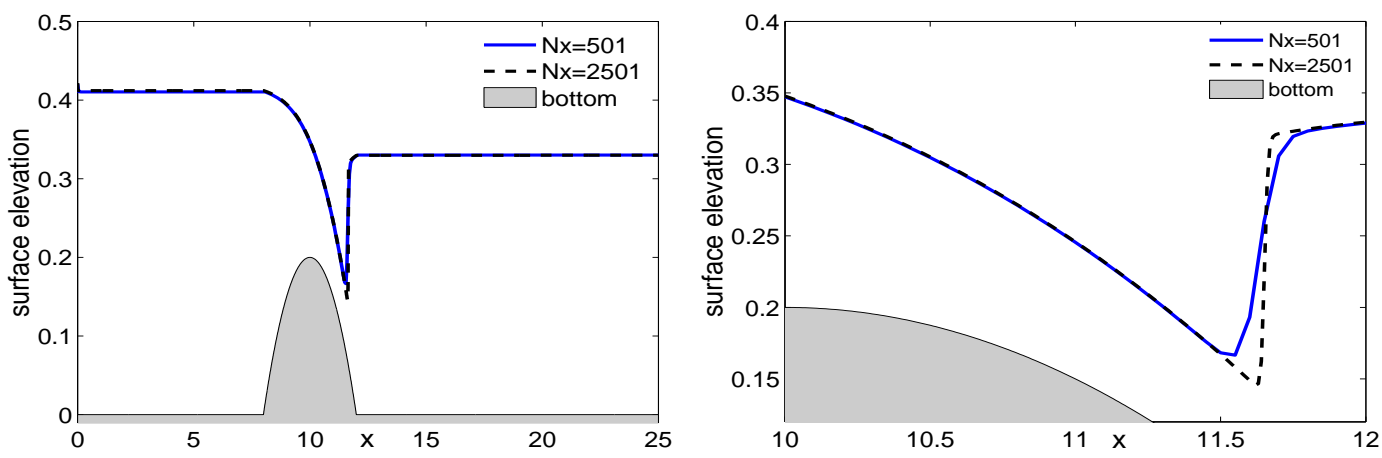

Figure 4: Transcritical flow with a shock. Left: Steady surface profile at time $t=200$ seconds. Right: Enlargement of the shock region.

\subsection{Dam break flow over a rectangular bump.}

We next present a dam break simulation above a rectangular bump, a rapidly varying flow over a discontinuous bottom topography. The computational domain is $0<x<1500$, and the initial conditions are

$$
h(x, 0)= \begin{cases}20, & x \leq 750 \\ 15, & \text { otherwise }\end{cases}
$$

and zero velocity. The bottom topography is assumed to be

$$
d(x)=\left\{\begin{aligned}
-8, & \text { if }|x-750| \leq 1500 / 8 \\
0, & \text { otherwise }
\end{aligned}\right.
$$

We adopted $\Delta x=3.75$ and $\Delta t=0.2$, and gravitational acceleration $g=9.81$, and the result is shown in Fig. 5. Thus as time progresses, a shock wave front travels to the right, 
while a rarefaction wave moves to the left. In the enlarged plot, our result is clearly comparable with results from Ref. [21], obtained using the non-oscillatory WENO scheme and Runge-Kutta Discontinuous Galerkin (RKGD) approach. Apart from small wiggles near the shock, our staggered scheme calculates the shock wave and its speed accurately. The staggered scheme is not monotone, and the small wiggles are due to dispersion error.
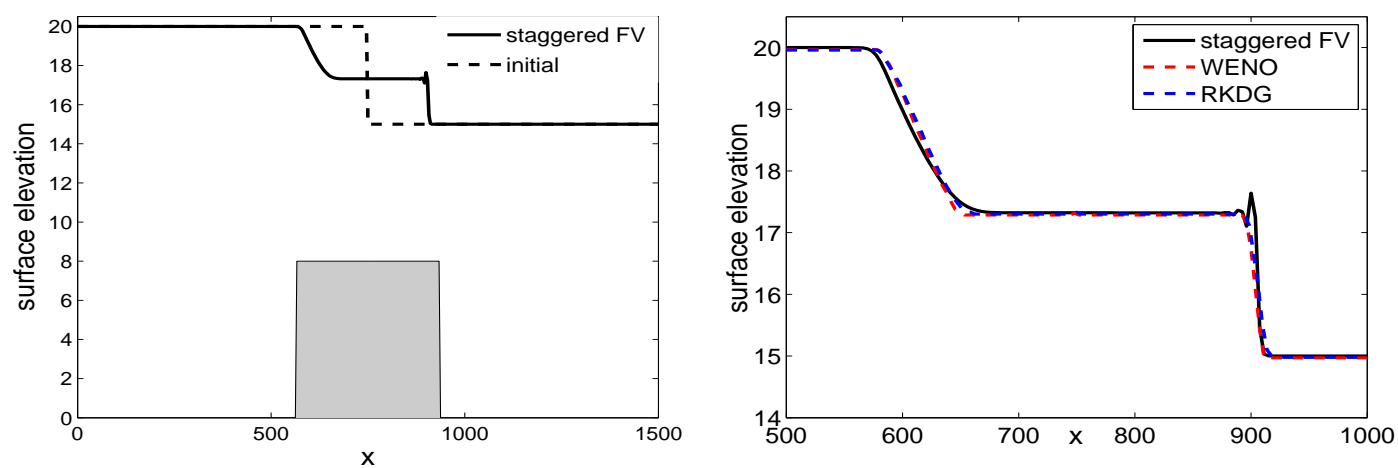

Figure 5: Dam break over a rectangular bump. Left: Surface profile at time $t=15 \mathrm{~s}$ compared with the initial profile. Right: Enlargement above the rectangular bump.

\subsection{Dam break into a dry bed.}

We also considered a dam break simulation involving a dry bed, where the computational domain was $-10<x<10$, the initial condition $h(x, 0)=1$ for $x<0$ and zero elsewhere, and zero initial velocity $u(x, 0)=0$. For this simulation, we used $\Delta x=0.01$ and $\Delta t=0.003$, gravitational acceleration $g=9.81$, and incorporated the wet-dry procedure. For a flat bed (no slope), we computed the surface elevation and horizontal velocity for two cases - with no friction and with friction. Fig. 6 displays our numerical results for the no friction case, in which the wave front propagates as a rarefaction wave, and the result from our momentum conservative scheme compares well with the exact solution. As observed by Stelling \& Duinmeijer [15], other conservative schemes (such as energyhead scheme) incorrectly produce a shock wave front, unlike our momentum conservative scheme for this dry bed case. Fig. 6 also shows that a frictional force with $C_{f}=0.1$ reduced the wave front location and velocity.

Further, we implemented the same problem for a sloping bed, where the depth $d(x)$ is a linear function with slope $S_{0}=0.01$. Fig. 7 illustrates our result, compared with the exact solution [5]

$$
h(x, t)= \begin{cases}1, & x<-t, \\ \frac{1}{9}\left(2+\frac{1}{2} S_{0} t-x / t\right)^{2}, & -t \leq x \leq x_{1}, \\ \max \left\{h_{s}(x, t),-d(x)\right\}, & x_{1} \leq x<x_{s}, \\ \max \{0,-d(x)\}, & x \geq x_{s},\end{cases}
$$



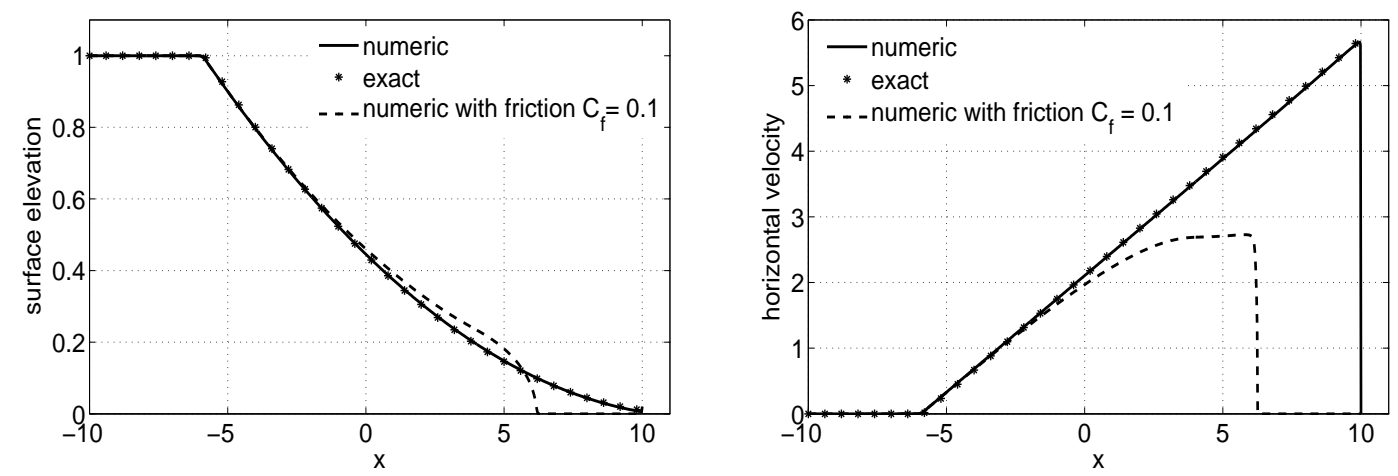

Figure 6: Dam break into a flat dry bed at time $t=1.8$ seconds, without friction and with friction coefficient $C_{f}=0.1$. Left: Water level $h$. Right: Horizontal velocity $u$.
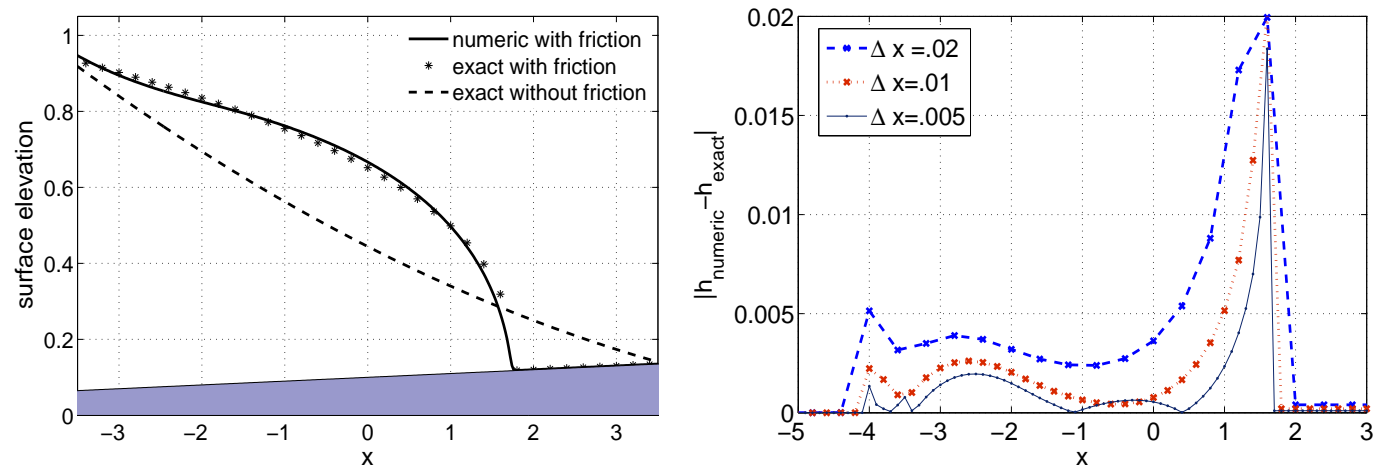

Figure 7: (Left) Surface profile in wave tip region. (Right) Difference between $h_{\text {numeric }}$ and $h_{\text {exact }}$ at time $t=4 \mathrm{~s}$ for various $\Delta x$.

with $h_{s}(x, t) \equiv\left[6 \alpha\left(x_{s}-x\right) u / R+\frac{1}{2} S_{0} t\right]^{1 / 3}$. The wave tip region is $x_{1}<x<x_{s}$ with $x_{1}=(3 u / 2-1) t$ and the wave front location is given by

$$
x_{s}=x_{1}+\frac{R}{u} \frac{1}{6 \alpha}\left(1-\frac{u}{2}+\frac{1}{2} S_{0} t\right)^{6} .
$$

For our simulation, we used the same initial data as before, but the gravitational acceleration $g$ was normalised to one. We took $\alpha=1$, and the Reynolds number $R e=4.73$ corresponding to friction parameter $C_{f}=5.7143$. As shown in Fig. 7 (Right), the error decreases as $\Delta x$ decreases, but there are rather large errors at the wave front location and a refinement does not reduce these errors significantly. Nevertheless, the agreement elsewhere between our simulation and the exact solution indicates that the momentum conservative scheme is otherwise suitable for this case. 


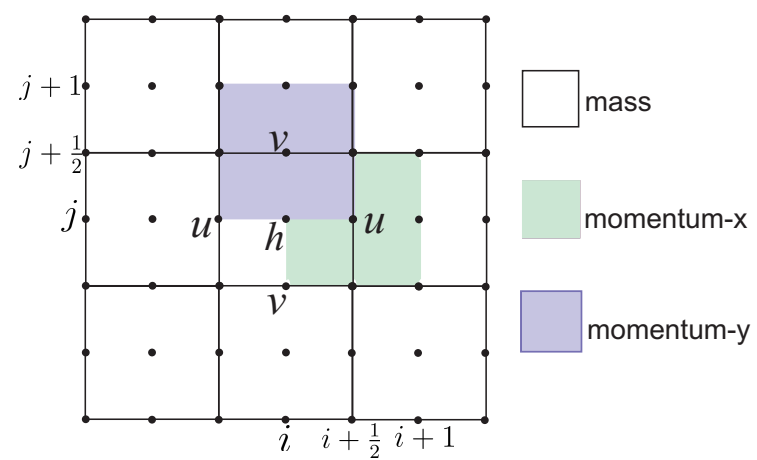

Figure 8: Arakawa- $C$ grid with cells for mass, momentum $-x$, and momentum $-y$.

\section{Finite Volume Method for 2D SWE Simulations}

In this section, a direct extension of the momentum conservative scheme to 2D shallow flow is discussed. This method is described in Ref. [15], but let us recall some details for clarity before we show that it is suitable for simulating wave run up on a conical island. The relevant 2D shallow water equations are

$$
\begin{aligned}
& h_{t}+(h u)_{x}+(h v)_{y}=0, \\
& \left.\left.{ }_{u} q_{t}+{ }_{u} q u+\frac{1}{2} g h^{2}\right)_{x}+{ }_{u} q v\right)_{y}=g h d_{x}, \\
& { }_{v} q_{t}+\left({ }_{v} q v+\frac{1}{2} g h^{2}\right)_{y}+\left({ }_{v} q u\right)_{x}=g h d_{y},
\end{aligned}
$$

where ${ }_{u} q=h u$ and ${ }_{v} q=h v$ denote the momentum in the $x$ and $y$ directions, respectively. The equivalent form are

$$
\begin{aligned}
& h_{t}+(h u)_{x}+(h v)_{y}=0, \\
& u_{t}+u u_{x}+v u_{y}+g \eta_{x}=0, \\
& v_{t}+u v_{x}+v v_{y}+g \eta_{y}=0,
\end{aligned}
$$

since the advection terms $u u_{x}, v u_{y}, u v_{x}, v v_{y}$ can be written in terms of ${ }_{u} q,{ }_{v} q, u, v$, and $h$ - i.e.

$$
\begin{aligned}
u u_{x}+v u_{y} & =\frac{1}{h}\left(\left({ }_{u} q u\right)_{x}-{ }_{u} q_{x} u\right)+\frac{1}{h}\left(\left({ }_{v} q u\right)_{y}-{ }_{v} q_{y} u\right), \\
u v_{x}+v v_{y} & \left.=\frac{1}{h}\left(\left({ }_{u} q v\right)_{x}-{ }_{u} q_{x} v\right)+\frac{1}{h}\left({ }_{v} q v\right)_{y}-{ }_{v} q_{y} v\right) .
\end{aligned}
$$

We considered the computational domain $[0, L] \times[0, M]$ with homogeneous Neumann boundary conditions along its four sides, and adopted the 2D staggered grid sketched in Fig. 8 that is a generalisation of the 1D staggered grid known as the Arakawa-C grid [1]. 
The analogous approximation of the continuity equation (5.4) at the mass cell around $\left(x_{i}, y_{j}\right)$ is

$$
\frac{d h_{i, j}}{d t}+\frac{{ }^{*} h_{i+1 / 2, j} u_{i+1 / 2, j}-{ }^{*} h_{i-1 / 2, j} u_{i-1 / 2, j}}{\Delta x}+\frac{{ }^{*} h_{i, j+1 / 2} v_{i, j+1 / 2}-{ }^{*} h_{i, j-1 / 2} v_{i, j-1 / 2}}{\Delta y}=0,
$$

with upwind approximations

$$
{ }^{*} h_{i+\frac{1}{2}, j}=\left\{\begin{array}{ll}
h_{i, j}, & \text { if } u_{i+1 / 2, j} \geq 0, \\
h_{i+1, j}, & \text { if } u_{i+1 / 2, j}<0,
\end{array} \quad * h_{i, j+\frac{1}{2}}= \begin{cases}h_{i, j}, & \text { if } v_{i, j+1 / 2} \geq 0 \\
h_{i, j+1}, & \text { if } v_{i, j+1 / 2}<0 .\end{cases}\right.
$$

The conservative approximation of the momentum equation (5.5) is implemented at the light grey cell around $\left(x_{i+1 / 2}, y_{j}\right)$, and (5.6) at the dark grey cell around $\left(x_{i}, y_{j+1 / 2}\right)$. Analogous to the $1 \mathrm{D}$ case, invoking the $2 \mathrm{D}$ momentum conservative approximation requires a consistent approximation for the advection terms in Eqns. (5.7) and (5.8). For positive flow directions $u>0, v>0$ they are

$$
\begin{aligned}
& \frac{d u_{i+1 / 2, j}}{d t}+\frac{\bar{u} \bar{q}_{i, j}}{\bar{h}_{i+1 / 2, j}} \frac{u_{i+1 / 2, j}-u_{i-1 / 2, j}}{\Delta x}+\frac{\bar{v} q_{i, j-1 / 2}}{\bar{h}_{i+1 / 2, j}} \frac{u_{i+1 / 2, j}-u_{i-1 / 2, j-1}}{\Delta y}+g \frac{\eta_{i+1, j}-\eta_{i, j}}{\Delta x}=0, \\
& \frac{d v_{i, j+1 / 2}}{d t}+\frac{\bar{u} q_{i-1 / 2, j}}{\bar{h}_{i, j+1 / 2}} \frac{v_{i, j+1 / 2}-v_{i-1, j+1 / 2}}{\Delta x}+\frac{\bar{v} q_{i, j}}{\bar{h}_{i, j+1 / 2}} \frac{v_{i, j+1 / 2}-v_{i, j-1 / 2}}{\Delta y}+g \frac{\eta_{i, j+1}-\eta_{i, j}}{\Delta y}=0,
\end{aligned}
$$

where $\bar{h}_{i+1 / 2, j}=\left(h_{i, j}+h_{i+1, j}\right) / 2, \bar{h}_{i, j+1 / 2}=\left(h_{i, j}+h_{i, j+1}\right) / 2$, and all other values are defined accordingly.

\section{Run Up Waves on a Conical Island}

We used our 2D numerical scheme in a context where large scale 2D experimental databases were available for comparison. Briggs et al. [3] conducted a laboratory experiment to investigate solitary wave run-up on a conical island. This experiment physically simulated a tsunami impact on a small conical island was simulated and the run-up height measured around the island.

The experimental setup involved a large basin, 25 metres long and 30 metres wide, with water depth $h=0.32$ metres and a small island. The centre of the island was located at $x=12.96$ and $y=13.80$ metres. The island was the shape of a truncated circular cone of diameter 7.2 metres at the toe and 2.2 metres at the crest, 0.625 metres high and with a side slope of $1: 4$. There were 27 gauges placed around the island to measure wave heights. Fig. 9 shows a simplified sketch of the experimental setup.

A solitary wave with amplitude $A / h=0.045$ was generated using the Directional Spectral Wave Generator (DSWG) from the left side. The origin of the $x$-axis of the coordinate system was located at the wave generator, and the $y$-axis was directed parallel to the wave generator. The run up wave around the conical island was measured at several positions, 
Table 1: Gauges and their positions.

\begin{tabular}{|c|c|c|}
\hline \hline Gauges & Direction & Position \\
\hline 6 & $0^{\circ}$ & $(9.36,13.80)$ \\
9 & $0^{\circ}$ & $(10.36,13.80)$ \\
16 & $90^{\circ}$ & $(12.96,11.22)$ \\
22 & $180^{\circ}$ & $(15.56,13.80)$ \\
\hline \hline
\end{tabular}

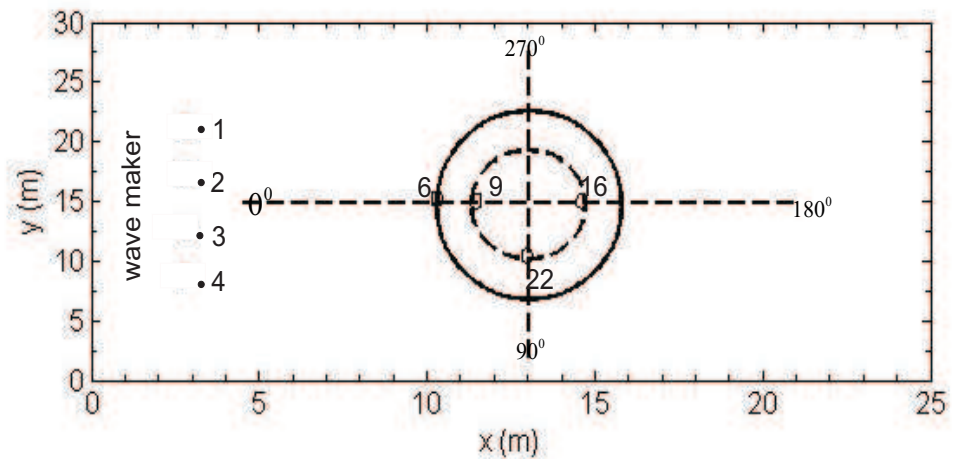

Figure 9: Top view of the experimental basin by Briggs et.al. [3].

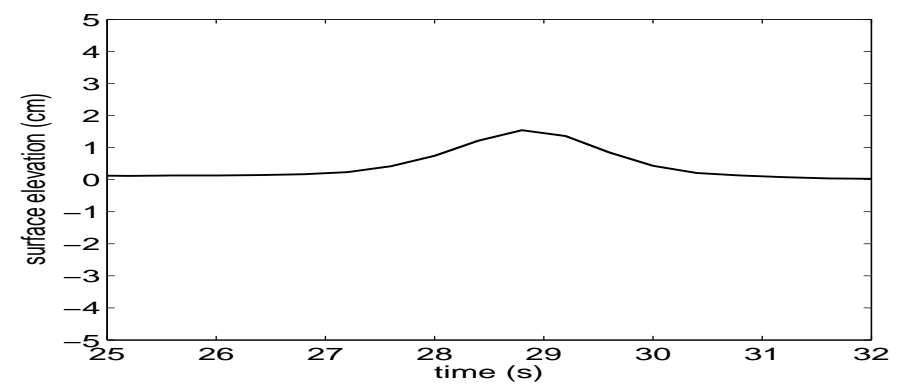

Figure 10: The incident solitary wave as recorded by gauge 3 .

and we compared the results from our 2D numerical scheme at four gauges as indicated in Fig. 9, with directions and positions given in Table 1.

The wave recorded by gauge 3, depicted in Fig. 10, is the generated solitary wave that we used as input for our numerical simulation. We implemented our 2D momentum conservative scheme for computing the surface elevation around a conical island, using $\Delta x=\Delta y=5$ centimetres and $\Delta t=0.01$ seconds. We recorded the surface profile at the four gauges positions above, and compare the results with experimental data of run-up height measurements from the NOAA Center for Tsunami Research, known as a benchmark for validating 2D run-up numerical models. The results plotted in Fig. 11 clearly show good agreement, so we again consider that the momentum conservative scheme is suitable for 2D wave run up simulations. 

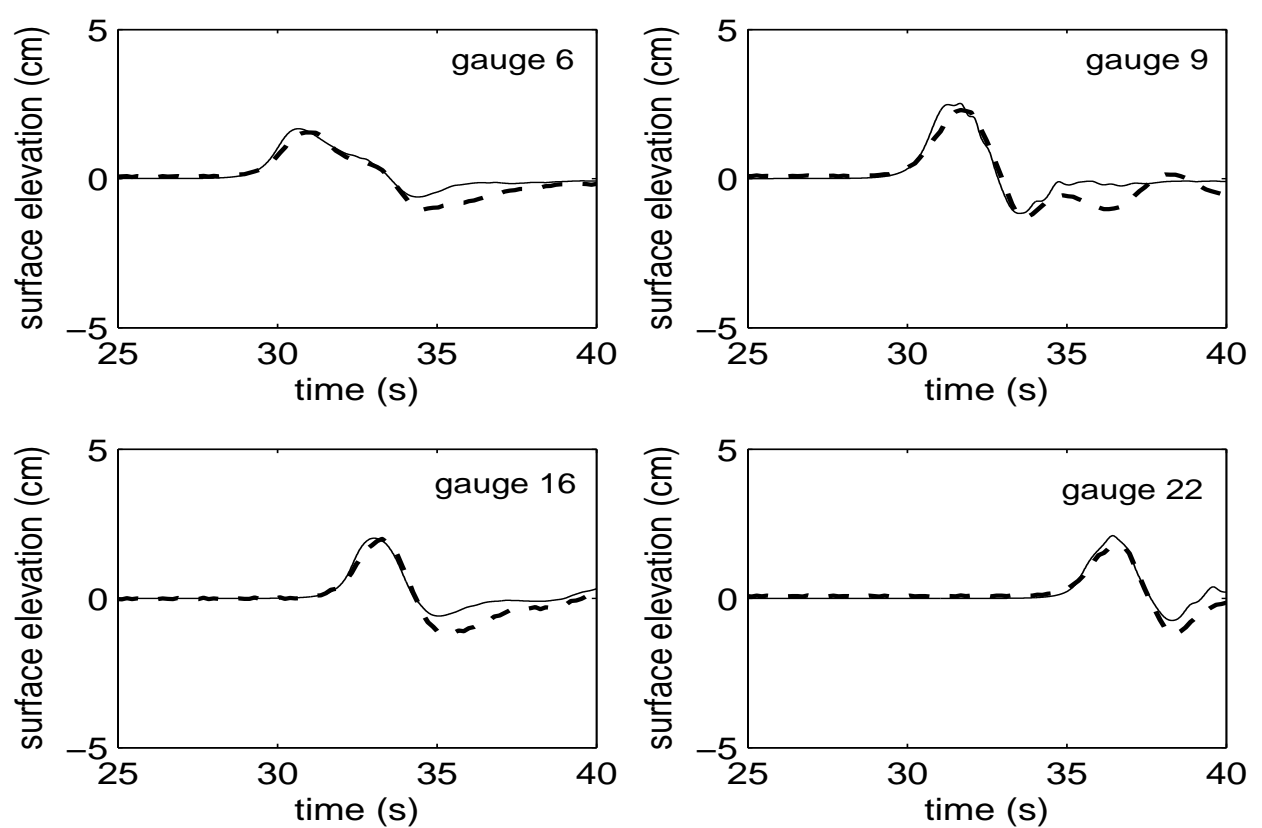

Figure 11: Surface elevations at gauges around a conical island, dash lines are experimental data and solid lines are numerical results.

\section{Conclusions}

We have implemented conservative approximations for solving both 1D and 2D shallow water equations, including a momentum conservative scheme was for approximating the nonlinear advection terms. Accurate results were obtained for the two 1D test cases of transcritical flow with shock and dam break flow over a rectangular bed. Indeed, good agreement with exact solutions were obtained for 1D dam break over a dry sloping bed, with and without friction. The computed 2D run up wave on a conical island was shown to be in good agreement with experimental data. These applications are benchmark problems for numerical solvers of the nonlinear shallow water equations, and it emerged that momentum conservative approximations are suitable for these problems. Moreover, no special treatment was required, indicating the robustness of this approach that is easy to implement.

\section{Acknowledgments}

Financial support from Riset KK 2014 is gratefully acknowledged. Our deep gratitude goes to Prof. Guus Stelling for introducing us to this very interesting and useful technique for fluid dynamics simulation, and we also thank Putu H. Gunawan for useful discussions. 


\section{References}

[1] A. Arakawa and V.R. Lamb, A potential enstrophy and energy conserving scheme for the shallow water equations. Monthly Weather Review 109, 18-36 (1981).

[2] F. Bouchut, Nonlinear Stability of Finite Volume Method for Hyperbolic Conservation Laws and Well-Balanced Schemes four Sources. Birkhauser Verlag, Germany, 2004.

[3] M.J. Briggs, C.E Synolakis, G.S. Harkins and D.R. Green, Laboratory experiments of tsunami runup on a circular island, Pure and Applied Geophysics 144, 569-593 (1995).

[4] V. Casulli, Semi-implicit finite difference method for three-dimensional shallow water flow, Int. J. Numer. Meth. Fluids 15, 169-148 (1992).

[5] H. Chanson, A simple solution of the laminar dam break wave, J. Appl. Fluid Mech. 1, 1-10 (2008).

[6] Q. Chen, J.T. Kirby, R.A. Dalrymple, A.B. Kennedy and A. Chawla, Boussinesq modeling of wave transformation, breaking, and runup. II: $2 \mathrm{D}$, J. Waterway, Port, Coastal, and Ocean Engineering, 126), 48-56 (2008.

[7] D. Doyen and P.H. Gunawan, Explicit Staggered Schemes for the Shallow Water Equations with Topography, in preparation.

[8] F.E. Ham, F.S. Lien and A.B. Strong, A fully conservative second order finite difference scheme for incompressible flow on nonuniform grids, J. Comp. Phys. 177, 117-133 (2002).

[9] K. Jochen, Ocean Modelling for Beginners. Springer-Heidelberg, Berlin Heidelberg, 2009.

[10] A. Kurganov and D. Levy, Central-upwind schemes for the Saint-Venant system, Math. Model. Numer. Anal. 36, 397- 425 (2002).

[11] R.J. LeVeque, Balancing source terms and flux gradients in high-resolution Godunov methods: the quasi-steady wave- propagation algorithm, J. Comp. Phys. 146, 346-365 (1998).

[12] P. Liu, Y.S. Cho, M.J. Briggs, U. Kanoglu and C.E. Synolakis, Runup of solitary wave on a circular island, J. Fluid Mech. 302, 259-285 (1995).

[13] Y. Morinishi, T.S. Lund, O.V. Vasilyev and P. Moin, Fully conservative higher order finite difference schemes for incompressible flow, J. Comp. Phys. 143, 90-124 (1998).

[14] S. Noelle, N. Pankratz, G. Puppo and J. R. Natvig, Well-balanced finite volume schemes of arbitrary order of accuracy for shallow water flows, J. Comp. Phys. 13, 474-499 (2006).

[15] G.S. Stelling and S.P.A. Duinmeijer, A staggered conservative scheme for every Froude number in rapidly varied shallow water flows, Int. J. Numer. Meth. Fluids 43, 1329-1354 (2003).

[16] G.S. Stelling and M. Zijlema, An accurate and efficient finite-difference algorithm for nonhydrostatic free-surface flow with application to wave propagation, Int. J. Numer. Meth. Fluids 43, 1-23 (2003).

[17] H. Z. Tang, T. Tang, and K. Xu, A gas-kinetic scheme for shallow-water equations with source terms, Z. Angew. Math. Phys. 55, 365-382 (2004).

[18] E.F. Toro, Riemann Solvers and Numerical Methods for Fluid Dynamics, A Practical Introduction, 3rd Edition. Springer Verlag, Berlin, (2009).

[19] O. Vasilyev, High order finite difference schemes on non-uniform meshes with good conservation properties, J. Comp. Phys, 157, ??? (2000).

[20] Y Wei, X.Z. Mao and K.F. Cheung, Well-balanced finite-volume model for long-wave runup, J. Waterway, Port, Coastal, and Ocean Engineering 132, 114-124 (2006).

[21] Y. Xing and C.W. Shu, A new approach of high order well-balanced finite volume WENO schemes and discontinuous Galerkin methods for a class of hyperbolic systems with source terms, Commun. Comput. Phys. 1, 100-134 (2006).

[22] M. Zijlema and G.S. Stelling, Efficient computation of surf zone waves using the nonlinear shallow water equations with non-hydrostatic pressure, Coastal Eng. 55, 780-790 (2008). 